\title{
Nôvo Phyllanthus (Euphorbiaceae) da Amazônia Brasileira
}

\author{
WILliam A. Rodrigues (*) \\ Instituto Nacional de Pesquisas \\ da Anazônia
}

\begin{abstract}
SINOPSE
Minucioso inventário florestal realizado por nós num trecho de mata de terra firme da Reserva Flo. restal Ducke permitiu-nos encontrar uma série interessante de plantas raras ou novas da Amazônia Bra sileira, entre elas a espécie abaixo, que admitimos ser nova para a Ciência.
\end{abstract}

Phyllanthus manausensis W. Rodrigues, n. sp. (Fig. 1).

Arbor $10 \mathrm{~m}$ alta $15 \mathrm{~cm}$ diametro, ramosa, ramificatione phyllanthoidea; ramuli ultimi foliati-florigeri decidui, teretes tenui, circiter 0,5mm diametro, glaberrimi, fusco-nigrescentes usque ad $18 \mathrm{~cm}$ longi foliola $7-13$ gerentes; petiolis usque ad $2 \mathrm{~mm}$ longis, modice caniculatis, in statu sicco nigrescentibus, glabris; stipulis triangulari-lanceolatis, subulatis, rigidulis, glabris paene $1 \mathrm{~mm}$ longis stramineis. Ramuli penultimi non-decidui, $2-3 \mathrm{~mm}$ điametro, cortice stramineo, glabro; juncturarum base ramulorum juniorum cataphyllis minutis obtecta. Foliorum lamina membranacea, ovata vel lanceolato-ovata glabra, discolor supra nigrescens, nitidula subtus pallidior, opaca usque ad $4,5 \mathrm{~cm}$ longa, $2,1 \mathrm{~cm}$ lata, basi rotundata vel obtusa, apice saepius acuta, margine integra; costa primaria supra impressa subtus prominula, costis secundariis utrinque prominulis tenuibus, saepissime alternis usque 12-jugis, adscendentibus, supra medium dichotomicis, venulis viz perspicuis reticulatis. Cymulae axillares biflorae, unisexuales, pedunculis microscopice pubescentibus, $2-3 \mathrm{~mm}$ longis; bracteis strobiliformibus minusculis. Flores feminei ca. $2 \mathrm{~mm}$ longi, pedicellis filiformibus ad $4-5 \mathrm{~mm}$ longis, glabris, nigrescentibus, apicem versus incras. satis; calycis laciniae 6 lanceolatae fusco-nigrescentes ca. $1,5 \mathrm{~mm}$ longae microscopice puberuli; discus annularis ca. $0,2 \mathrm{~mm}$ altus; ovarium subglobosum vel ovoideum $1 \mathrm{~mm}$ diametro, glabrum; sty]i 3 , ca. $0,5 \mathrm{~mm}$ longi, glabri, subsessiles, reflexi, lanceolati, ad basin connati. Capsula immatura subglobosa ca. ... 7-8mm diametro, glabra, sessilis, virens, in statu sicco nigra; semina triangularia, fusca laevia, ca. $5 \mathrm{~mm}$ longa, $2 \mathrm{~mm}$ lata. Flores masculi hucusque ignoti.

Habitat: in silva terrae altae vicinia Manaus, Amazonas, Brasília, ubi nonihil rara.

Holotypus: W. A. Rodrigues, 7520 (INPA 16822), 18-X-1963; Reserva Florestal Ducke, árvore $\mathrm{X}-325$ do inventário florestal; árvore de $10 \mathrm{~m}$ de altura por $11 \mathrm{~cm}$ de diâmetro, rara na mata de terra firme, solo argiloso. (Isotypus NY) .

Paratypus: G. T. Prance, B. S. Pena \& J. F. Ramos, 3825 (INPA 19449); road Ma-

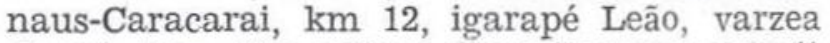
forest; tree, $10 \mathrm{~m} \times 15 \mathrm{~cm}$ diameter; young fruit green.

Em virtude de as flôres masculinas não serem conhecidas até agora, torna-se difícil estabelecer a posição taxonômica da presente €spécie dentro do gênero.

Submetida a espécie em questão ao Dr. Grady L. Webster, êste em carta pessoal é de opiniấo seguinte: "The plant seems to belong in a small section which includes $P$. rupestris HBK., $P$. adiantoides $\mathrm{Kl}$., and $P$. spruceanus Muell. Arg. It does not fit any of those species, however".

\section{S U M M A R Y}

The author describes in this paper a new species of the family Euphorbiaceae (Plyyllanthus manaitsensis W. Rodrigues, $\mathrm{n} . \mathrm{sp}$ ), a relatively rare tree of the upland forest near Manaus, in the brazilian State of Amazonas.

\section{B I B L I O G R A F I A}

JABLONSKI, E.

1967 - Euphorbiaceae. In: The Botany of the Guyana Highland. Part VII. Mem. N. Y. Bot. Garden, 17(1) : 81-118.

MUELLER, J .

1866 - Euphorbiaceae - Phyllantheae. DC. Prodr., $15(2): 215-491$.

1873 - Euphorbiaceae - Euphyllantheae. In : MARTrUS, C. F. P. von - Flora Brasiliensis, 11(2):23-75.

\footnotetext{
(*) - Bolsista do Conselho Nacional de Pesquisas.
} 


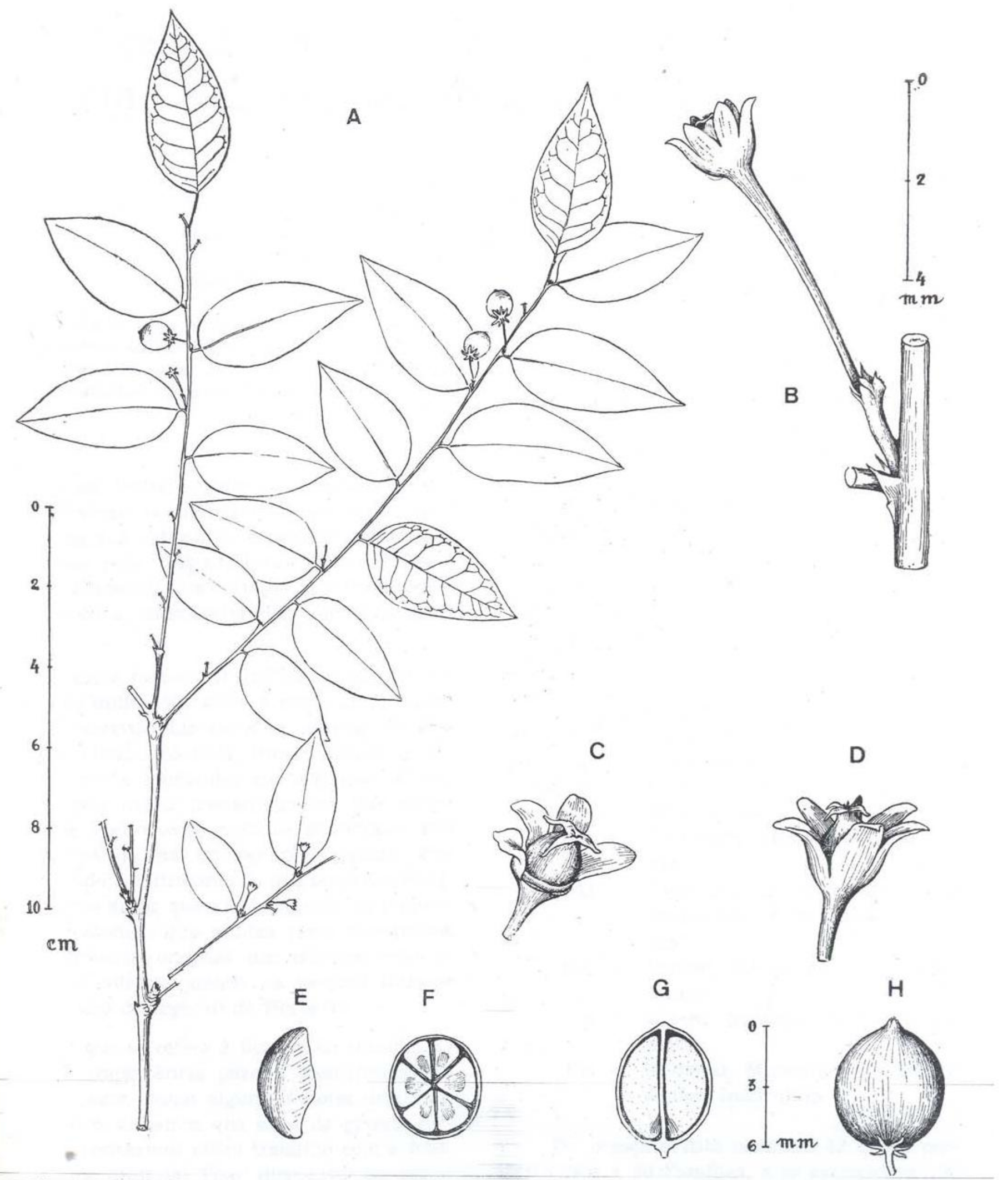

Fig. 1 - Phyllanthus manausensis W. Rodrigues, n. sp. A, Hábito do raminho florífero. $B$, Inflorescência feminina; $C$ e $D$, Flor feminina; $E$, Semente vista de perfil; $F$, Seção transversal do fruto; $G$, Fruto em secção longitudinal; $H$, Fruto inteiro. (Desenhos de J. Dellome Filho). 